\title{
Anti-CTLA-4/LAG-3 Bispecific Antibody XmAb22841
}

National Cancer Institute

\section{Source}

National Cancer Institute. Anti-CTLA-4/LAG-3 Bispecific Antibody XmAb22841. NCI

Thesaurus. Code C159530.

An Fc-eng ineered bispecific antibody directed against the human negative

immunoregulatory checkpoint receptors cytotoxic T-lymphocyte-associated antigen 4 (CT LA4; CT LA-4) and lymphocyte activation gene 3 protein (LAG3; LAG-3; CD223), with potential immune checkpoint inhibitory and antineoplastic activities. Upon administration, anti-CT LA-4/LAG-3 bispecific antibody XmAb22841 targets and binds to both CT LA-4 and LAG-3 expressed on T-cells in the tumor microenvironment (TME). Both CT LA-4 and LAG-3 are inhibitory receptors and members of the immunoglobulin superfamily (IgSF); they are overexpressed by regulatory T-cells (Tregs) in the TME where they downregulate T-cell activation and proliferation. Dual checkpoint blockade of CT LA-4 and LAG-3 with XmAb22841 may enhance T-cell activation and proliferation more than the blockade of either immune checkpoint receptor alone. The eng ineered Fc domain increases the stability and half-life of the antibody. 